\title{
Observaton of tunneling of slow and fast electromagnetic modes in coupled periodic waveguides
}

Ha, Sangwoo; Sukhorukov, Andrey A.; Lavrinenko, Andrei; Shadrivov, llya V.; Powell, David A.; Kivshar, Yuri S.

Published in:

Applied Physics Letters

Link to article, DOI:

$10.1063 / 1.3553785$

Publication date:

2011

Document Version

Publisher's PDF, also known as Version of record

Link back to DTU Orbit

Citation (APA):

Ha, S., Sukhorukov, A. A., Lavrinenko, A., Shadrivov, I. V., Powell, D. A., \& Kivshar, Y. S. (2011). Observaton of tunneling of slow and fast electromagnetic modes in coupled periodic waveguides. Applied Physics Letters, 98(6), 061909. https://doi.org/10.1063/1.3553785

\section{General rights}

Copyright and moral rights for the publications made accessible in the public portal are retained by the authors and/or other copyright owners and it is a condition of accessing publications that users recognise and abide by the legal requirements associated with these rights.

- Users may download and print one copy of any publication from the public portal for the purpose of private study or research.

- You may not further distribute the material or use it for any profit-making activity or commercial gain

- You may freely distribute the URL identifying the publication in the public portal 


\title{
Observation of tunneling of slow and fast electromagnetic modes in coupled periodic waveguides
}

\author{
Sangwoo Ha, ${ }^{1}$ Andrey A. Sukhorukov, ${ }_{1}^{1, a)}$ Andrei V. Lavrinenko, ${ }^{2,1}$ Ilya V. Shadrivov, ${ }^{1}$ \\ David A. Powell, ${ }^{1}$ and Yuri S. Kivshar ${ }^{1}$ \\ ${ }^{1}$ Nonlinear Physics Centre, Research School of Physics and Engineering, Australian National University, \\ Canberra, Australian Capital Territory 0200, Australia \\ ${ }^{2}$ DTU Fotonik, Department of Photonics Engineering, Technical University of Denmark, \\ DK-2800 Kgs Lyngby, Denmark
}

(Received 1 July 2010; accepted 21 January 2011; published online 9 February 2011)

\begin{abstract}
We report the experimental observation of tunneling of slow and fast electromagnetic modes in coupled periodic waveguides shifted longitudinally by half of modulation period. According to the symmetry analysis, such a coupler supports two electromagnetic modes with exactly matched slow or fast group velocities but different phase velocities for frequencies close to the edge of the photonic band. We confirm the predicted properties of the modes by directly extracting their dispersion and group velocities from the near-field measurements using specialized Bloch-wave spectral analysis method. (C) 2011 American Institute of Physics. [doi:10.1063/1.3553785]
\end{abstract}

The physics and applications of slow electromagnetic modes are attracting increasing attention in recent years, including advances in slow-light manipulation. ${ }^{1}$ One potential application of velocity control in optical spectral range is for delay lines, enabling all-optical pulse multiplexing and synchronization in communication networks. Another remarkable advantage of the slow-light regime is related to the enhancement of light-matter interactions, where very small changes of medium characteristics can significantly affect the light propagation. Enhanced interactions between multiple optical waves may be realized if their velocities are reduced simultaneously, including all-optical switching of slow light in the form of gap solitons. ${ }^{2}$

In this letter, we demonstrate experimentally simultaneous realization of slow group velocities in the same frequency region for the electromagnetic supermodes of two side-coupled periodic waveguides, when the waveguides are shifted longitudinally by half of the modulation period in agreement with our theoretical predictions. ${ }^{3}$ Such structures can be used to perform spatial switching of slow electromagnetic waves through the beating of modes, which is registered in experiment as tunneling between the coupled waveguides. Moreover, we show that the tunneling also persists in the regime of fast group velocities.

For our experimental studies, we use arrays of dielectric rods, ${ }^{3,4}$ and design the periodic waveguides to realize the slow group velocities at microwave frequencies. This enables us to perform faster structure prototyping and obtain detailed measurements including near-field profiles which is important for the exploration of fundamental effects. Indeed, previous experiments performed at microwave frequencies ${ }^{5-7}$ have facilitated the investigation of tunneling of electromagnetic modes between waveguides and cavities before the realization of these effects in optics. The effect of tunneling at slow group velocities demonstrated in this work would also occur in various types of side-coupled periodic waveguides operating at optical wavelengths including Bragg gratings, ${ }^{2,8-10}$ photonic crystals, ${ }^{11,12}$ and coupled-rod

\footnotetext{
${ }^{a)}$ Electronic mail: ans124@physics.anu.edu.au.
}

waveguides ${ }^{13-16}$ since the effect of longitudinal shift on dispersion of slow modes is generic according to the symmetry and perturbation theory analysis. ${ }^{3,12}$

We fabricate periodic waveguides using dielectric pillars made from alumina with a height of $1 \mathrm{~cm}$, a diameter of $4.18 \mathrm{~mm}$, and a refractive index of 2.5. The rods are inserted in holes drilled in a polystyrene foam, which acts as practically transparent substrate material at microwave frequencies around $10 \mathrm{GHz}$ where its refractive index value is $n \simeq 1.01$. The polystyrene frame is drilled using a computer-controlled 3-D milling machine (Roland DG MDX-40) to produce precise periodicity and longitudinal shift between the waveguides. The individual waveguides consist of 20 pillars with a period of $d=14 \mathrm{~mm}$, and their transverse separation is $14 \mathrm{~mm}$. We fabricate two types of coupled waveguide configurations: symmetric, when there is no longitudinal shift between the waveguides (Fig. 1, top), and antisymmetric,
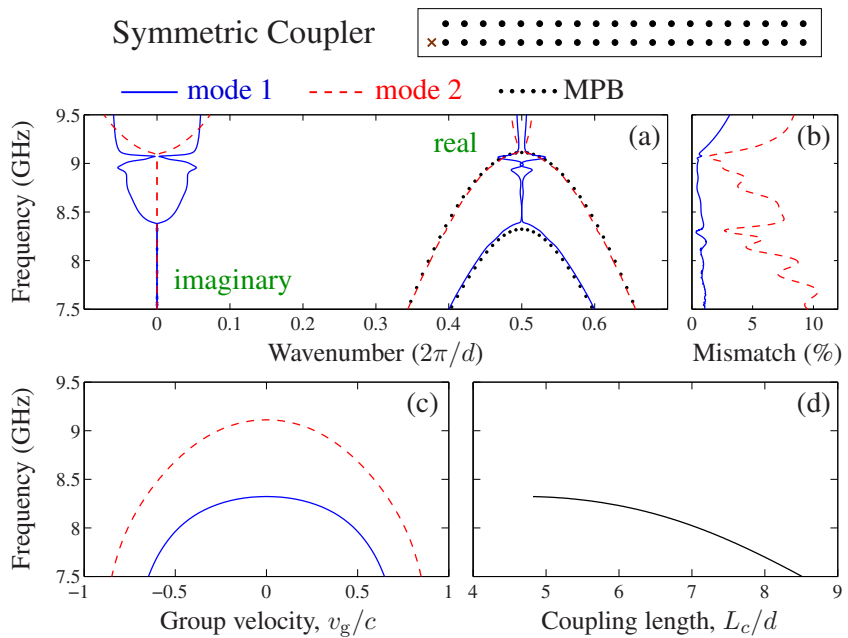

FIG. 1. (Color online) Top: sketch of symmetric coupler; dots indicate rods, cross indicates source antenna. (a) Experimentally extracted dispersion of two fundamental modes (1: solid, 2: dashed lines) and 2D MPB numerical simulations with effective refractive index $n=2.38$ (dotted lines). (b) Mismatch between the extracted modes and the symmetric and antisymmetric parts of the measured field. (c) Group velocity and (d) coupling length calculated with MPB. 


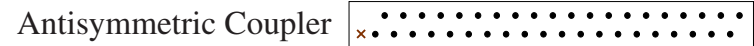
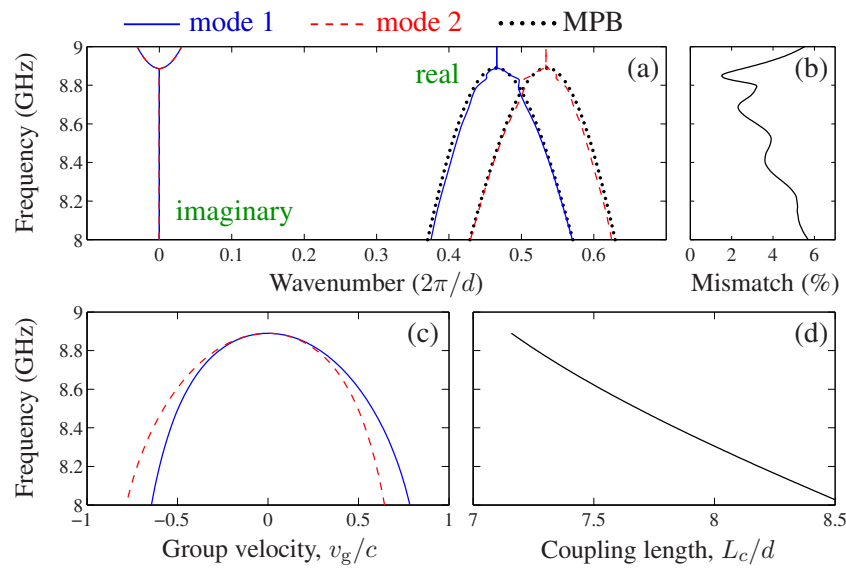

FIG. 2. (Color online) Results for the antisymmetric coupler. Notations are the same as in Fig. 1, except that effective refractive index in simulations is $n=2.32$ and (b) shows a mismatch between extracted modes superposition and the measured field.

when the longitudinal shift is equal to half of the modulation period (Fig. 2, top).

The structure characterization is performed using a setup which has been previously developed to study various photonic crystal designs with microwaves, see Fig. 2 in Ref. 17. The fabricated waveguides are sandwiched between two parallel metal plates separated by approximately $11 \mathrm{~mm}$. Also placed between the plates is the carbon impregnated foam-a sawtooth-shaped absorber that suppresses reflections from the edge of the metal plates. The $20 \mathrm{GHz}$ vector network analyzer (VNA) (Rohde and Schwarz ZBV20) is used to measure the amplitude and phase of the field, with the monopole antennas connected to the VNA providing tunable $\mathrm{cw}$ source and detector. At the operating frequency range, only vertically polarized TEM mode is supported. The detecting antenna is attached to the upper metal plate that was fixed in place, while the source antenna is attached to the base plate (and the waveguide) that is mounted on the translation stage. In this way, near-field data of the electric field can be collected over the whole waveguide area from above (we use $1 \mathrm{~mm}$ resolution for our experiment).

We process the near-field data $E(x, z)$ measured at a set of different frequencies to extract the dispersion of modes of the coupled periodic waveguides. Due to periodicity, the mode profiles satisfy the Floquet-Bloch symmetry condition, and we can apply specially developed high-resolution spectral analysis method by performing least squares optimization accounting for the symmetry constraints. ${ }^{18}$ In performing the spectral analysis, we additionally employ the symmetry properties. In particular, for a symmetric coupler the modes have either odd or even symmetry and we separate their profiles as $E_{\text {even }}(x, z)=E(x, z)+E(-x, z)$ and $E_{\text {odd }}(x, z)$ $=E(x, z)-E(-x, z)$. For the antisymmetric coupler, however, the modes are neither even nor odd ${ }^{11}$ and must be extracted simultaneously. We exploit a symmetry of the structure with respect to a "glide plane" transformation which combines translation and reflection $(x, z) \rightarrow(-x, z+d / 2)$ to apply the general extraction procedure. ${ }^{18}$

We are then able to extract dispersion curves of frequency versus wavenumber directly from the experimental data, and the results for the two fundamental supermodes of the symmetric and antisymmetric coupled waveguides are

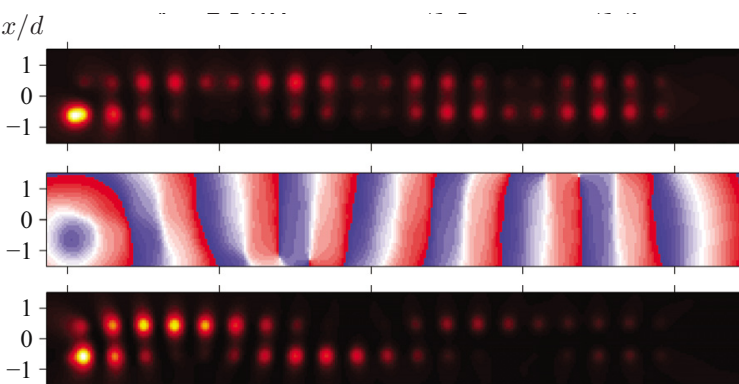

(b)
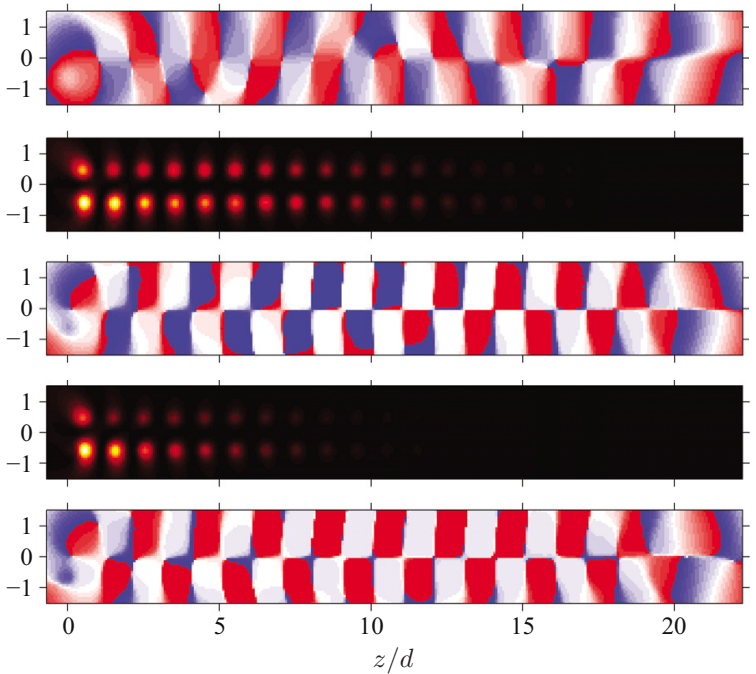

FIG. 3. (Color online) [(a)-(d)] Experimental intensity (top subplots) and phase (bottom subplots) profiles of near-field electric field measurements for the symmetric coupler at frequencies of (a) 7.5, (b) 8.38, (c) 9.11, and (d) $9.16 \mathrm{GHz}$. The corresponding group velocities of propagating modes according to Fig. 1(c) are (a) $c / 1.5$ (mode 1) and $c / 1.2$ (mode 2), (b) $c / 9$ (mode 1) and $c / 1.6$ (mode 2), and (c) $c / 29$ (mode 2).

presented in Figs. 1(a) and 2(a), respectively. Most importantly, we determine both the real and imaginary (for evanescent waves) parts, enabling full characterization of both the propagating and evanescent modes. We check that the extraction accuracy is good, as indicated by the low mismatch plotted in Figs. 1(b) and 2(b). The irregular behavior of mode 1 dispersion for a symmetric coupler around $9.1 \mathrm{GHz}$ is due to a resonant enhancement of mode 2 amplitude at slow group velocity, such that extraction for much weaker mode 1 becomes less accurate. The mismatch starts to increase as the frequency is tuned further into the bandgap (higher frequencies), as the evanescent mode intensities decay quickly inside the structure and noise due to nonguided waves which are radiated sideways starts to affect more strongly the extraction results.

We also calculate numerically the modal dispersion of an equivalent two-dimensional (2D) photonic structure with an effective refractive index, using the freely available MPB software package, ${ }^{19}$ which provides dispersion for propagating modes with real-valued wavenumbers. The real parts of the dispersion curves extracted from experiment agree well with the calculations shown as the dotted curves in Figs. 1(a) and 2(a). Additionally, the growth of imaginary parts indicating the formation of evanescent modes coincides well with the mode cut-off and bandgap frequencies. We use the calculated dispersion dependencies to determine the group velocities of the guided modes. We observe that for a symmetric coupler (Fig. 1), the slow-velocity regime is achieved at different frequencies. On the contrary, the group velocities of 
$x / d$

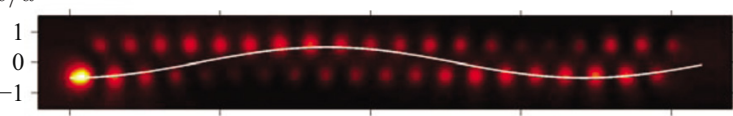

(a)

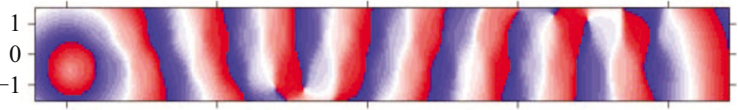

(b)
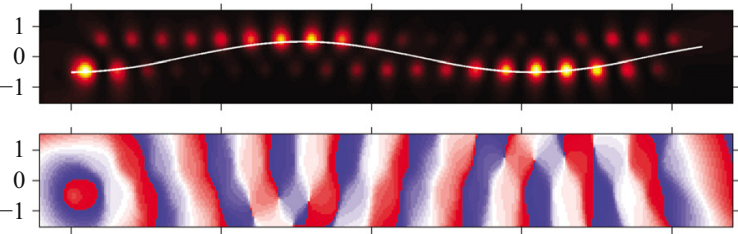

(c)
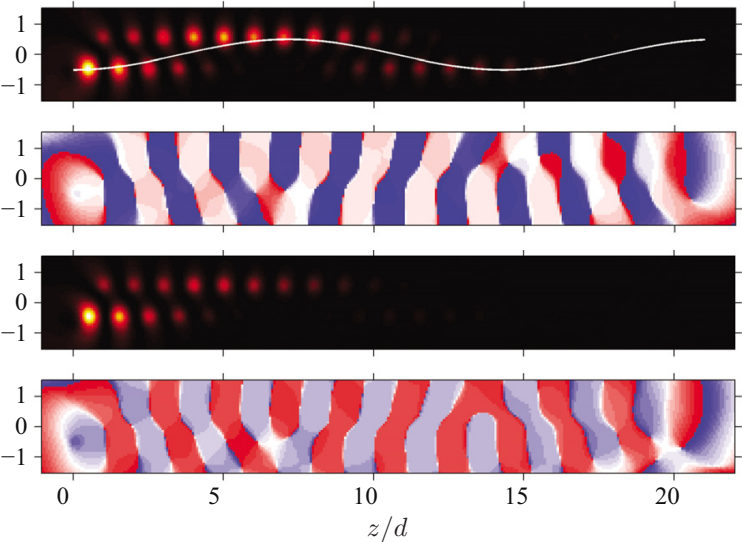

FIG. 4. (Color online) [(a)-(d)] Experimental intensity (top subplots) and phase (bottom subplots) profiles of the antisymmetric coupler at frequencies of (a) 8, (b) 8.5, (c) 8.885, and (d) $8.95 \mathrm{GHz}$. The corresponding group velocities of propagating modes according to Fig. 1(c) are (a) $c / 1.3$ (mode 1) and $c / 1.6$ (mode 2), (b) $c / 1.7$ (mode 1$)$ and $c / 2$ (mode 2), and (c) $c / 15$ (modes 1 and 2).

two modes are exactly matched for the antisymmetric coupler [Fig. 2(c)]. These results agree with the theoretical perturbation theory analysis. ${ }^{3}$ We also perform finite-difference time-domain (FDTD) simulations, which confirm the same features of pulse propagation dynamics as reported in Ref. 3 .

We also observe a clear difference between the symmetric and antisymmetric couplers by directly analyzing the electric field profiles, see Figs. 3 and 4. When the frequency is tuned far from the band-edge, the symmetric and antisymmetric waveguides show similar characteristics, as the wave propagates along a sinusoidal trajectory at group velocities compared to speed of light in vacuum. Mathematically, such periodic tunneling between the waveguides arises due to the beating of the two supermodes. The beating period, or the coupling length, can be determined as a propagation distance where the relative phase between the modes changes by $\pi$, and we plot the corresponding dependencies of the coupling length on frequency in Figs. 1(d) and 2(d), respectively.

When the frequency is tuned close to the cut-off of mode 1 in the symmetric waveguide at $f=8.38 \mathrm{GHz}$, the mode 1 slows down significantly while the group velocity of mode 2 is still large. Spatial mode beating is still observed; however, the two modes have significantly different group velocities, which would lead to pulse break-up according to FDTD simulations. ${ }^{3}$ As the frequency is further increased, the mode 2 approaches slow-velocity regime near the band-edge $(f$ $=9.11 \mathrm{GHz}$ ); however, the spatial switching disappears since mode 1 is evanescent and cannot propagate through the structure.
In the antisymmetric coupler, however, we observe that the periodic tunneling is maintained as the frequency is tuned toward the band-edge, and it occurs for slow modes near the band-edge $(f=8.885 \mathrm{GHz})$ where $V_{g 1} \simeq V_{g 2} \simeq c / 15$. Additionally, we note that the coupling length changes weakly as the group velocity value changes by orders of magnitude close to the band-edge, cf. Figs. 1(c) and 1(d), and this would enable complete tunneling of slow pulses between the waveguides as shown in FDTD modeling. ${ }^{3}$ We observe that the tunneling between the waveguides is maintained even as the frequency is tuned inside the bandgap at $f=8.9 \mathrm{GHz}$. This happens because the frequency is close to the bandgap edge and the evanescent decay length is longer than the mode beating length. It is interesting to note that for evanescent modes the group velocity can become formally infinite, corresponding to propagation faster than the speed of light. ${ }^{20}$

In conclusion, we have studied experimentally the tunneling of slow and fast electromagnetic modes in coupled periodic waveguides. Using the near-field measurements of field profiles and characterizing dispersion dependencies, we have observed a key difference between symmetric and antisymmetric periodic structures. We have demonstrated that only in the latter case, when two modes which group velocities are matched, tunneling of slow and fast modes between the periodic waveguides becomes possible. We anticipate that future studies may demonstrate flexible spatiotemporal manipulation of light by operating the coupled waveguides in tunable and nonlinear regimes at optical wavelengths.

This work was supported by the Australian Research Council.

${ }^{1}$ Slow Light: Science and Applications, edited by J. B. Khurgin and R. S. Tucker (Tailor \& Francis, New York, 2009).

${ }^{2}$ S. Ha and A. A. Sukhorukov, J. Opt. Soc. Am. B 25, C15 (2008).

${ }^{3}$ A. A. Sukhorukov, A. V. Lavrinenko, D. N. Chigrin, D. E. Pelinovsky, and Yu. S. Kivshar, J. Opt. Soc. Am. B 25, C65 (2008).

${ }^{4}$ D. N. Chigrin, A. V. Lavrinenko, and C. M. S. Torres, Opt. Express 12, 617 (2004).

${ }^{5}$ M. Bayindir and E. Ozbay, Appl. Phys. Lett. 81, 4514 (2002).

${ }^{6}$ P. Kohli, C. Christensen, J. Muehlmeier, R. Biswas, G. Tuttle, and K. M. Ho, Appl. Phys. Lett. 89, 231103 (2006).

${ }^{7}$ R. J. Liu, Z. Y. Li, Z. F. Feng, B. Y. Cheng, and D. Z. Zhang, J. Appl. Phys. 103, 094514 (2008).

${ }^{8}$ R. März and H. P. Nolting, Opt. Quantum Electron. 19, 273 (1987).

${ }^{9}$ M. Åslund, J. Canning, L. Poladian, C. M. de Sterke, and A. Judge, Appl. Opt. 42, 6578 (2003)

${ }^{10}$ J. M. Castro, D. F. Geraghty, S. Honkanen, C. M. Greiner, D. Iazikov, and T. W. Mossberg, Opt. Express 13, 4180 (2005).

${ }^{11}$ H. Benisty, J. Appl. Phys. 79, 7483 (1996).

${ }^{12}$ S. W. Ha, A. A. Sukhorukov, K. B. Dossou, L. C. Botten, A. V. Lavrinenko, D. N. Chigrin, and Yu. S. Kivshar, Opt. Express 16, 1104 (2008).

${ }^{13}$ M. Tokushima, H. Yamada, and Y. Arakawa, Appl. Phys. Lett. 84, 4298 (2004).

${ }^{14}$ S. Assefa, P. T. Rakich, P. Bienstman, S. G. Johnson, G. S. Petrich, J. D. Joannopoulos, L. A. Kolodziejski, E. P. Ippen, and H. I. Smith, Appl. Phys. Lett. 85, 6110 (2004).

${ }^{15}$ X. Y. Ao, L. Liu, L. Wosinski, and S. L. He, Appl. Phys. Lett. 89, 171115 (2006).

${ }^{16}$ J. She, E. Forsberg, X. Y. Ao, and S. L. He, J. Opt. A, Pure Appl. Opt. 8, 345 (2006).

${ }^{17}$ A. Matthews and Y. Kivshar, Appl. Phys. Lett. 93, 131901 (2008).

${ }^{18}$ S. Ha, A. A. Sukhorukov, K. B. Dossou, L. C. Botten, C. M. de Sterke, and Yu. S. Kivshar, Opt. Lett. 34, 3776 (2009).

${ }^{19}$ S. G. Johnson and J. D. Joannopoulos, Opt. Express 8, 173 (2001).

${ }^{20}$ H. G. Winful, Phys. Rep. 436, 1 (2006). 